\title{
BMJ Global Health Prevalence of substandard and falsified artemisinin-based combination antimalarial medicines on Bioko Island, Equatorial Guinea
} Harparkash Kaur, ${ }^{1}$ Elizabeth Louise Allan, ${ }^{1}$ Ibrahim Mamadu, ${ }^{1}$ Zoe Hall, ${ }^{1}$
Michael D Green, ${ }^{2}$ Isabel Swamidos, ${ }^{2}$ Prabha Dwivedi, ${ }^{3}$ Maria Julia Culzoni, ${ }^{4}$
Facundo M Fernandez, ${ }^{5}$ Guillermo Garcia, ${ }^{6}$ Dianna Hergott, ${ }^{6}$ Feliciano Monti ${ }^{6}$

\section{ABSTRACT}

Introduction Poor-quality artemisinin-containing antimalarials (ACAs), including falsified and substandard formulations, pose serious health concerns in malaria endemic countries. They can harm patients, contribute to the rise in drug resistance and increase the public's mistrust of health systems. Systematic assessment of drug quality is needed to gain knowledge on the prevalence of the problem, to provide Ministries of Health with evidence on which local regulators can take action.

Methods We used three sampling approaches to purchase 677 ACAs from 278 outlets on Bioko Island, Equatorial Guinea as follows: convenience survey using mystery client ( $n=16$ outlets, 31 samples), full island-wide survey using mystery client $(n=174$ outlets, 368 samples) and randomised survey using an overt sampling approach ( $\mathrm{n}=88$ outlets, 278 samples). The stated active pharmaceutical ingredients (SAPIs) were assessed using high-performance liquid chromatography and confirmed by mass spectrometry at three independent laboratories.

Results Content analysis showed $91.0 \%$ of ACAs were of acceptable quality, $1.6 \%$ were substandard and $7.4 \%$ falsified. No degraded medicines were detected. The prevalence of medicines without the SAPIs was higher for ACAs purchased in the convenience survey compared with the estimates obtained using the full island-wide survey-mystery client and randomised-overt sampling approaches. Comparable results were obtained for full island survey-mystery client and randomised overt. However, the availability of purchased artesunate monotherapies differed substantially according to the sampling approach used (convenience, $45.2 \%$; full island-wide survey-mystery client, $32.6 \%$; random-overt sampling approach, $21.9 \%$ ). Of concern is that $37.1 \%$ $(n=62)$ of these were falsified.

Conclusion Falsified ACAs were found on Bioko Island, with the prevalence ranging between $6.1 \%$ and $16.1 \%$, depending on the sampling method used. These findings underscore the vital need for national authorities to track the scale of ineffective medicines that jeopardise treatment of life-threatening diseases and value of a representative sampling approach to obtain/measure the true prevalence of poor-quality medicines.

\section{INTRODUCTION}

Malaria can be treated, provided access to good quality, efficacious medicines is available. ${ }^{12}$ Plasmodium falciparum parasites cause the deadliest form of malaria, which in 2015 led to 212 million malaria cases and resulted in 429000 deaths. ${ }^{3}$ The WHO recommends artemisinin combination therapies (ACTs) as first-line treatment, ${ }^{4}$ adopted in 81 countries by the end of 2014 and shown to have greatly contributed to the reduction in malaria morbidity and mortality. ${ }^{5}$ Yet poor-quality ACTs (falsified/substandard, unregistered and degraded) pose an enormous threat to patients who are malaria-infected and may lead to drug resistance. Protecting the efficacy of ACTs has recently been declared a global public health priority by WHO. ${ }^{6}$

Falsified monotherapies and ACTs have been reported in Africa, where there is a high burden of potentially fatal falciparum malaria. $^{7}$ In 2011, we reported the case of a Spanish traveller whose life was endangered after taking artesunate monotherapy blister packed tablets, purchased in Bata, mainland Equatorial Guinea, which were falsified as they did not contain the stated active pharmaceutical ingredient (SAPI), demonstrating the threat from counterfeit medicines in this region. ${ }^{8}$ All medicines imported into the country (by donors, government or private sector) require the authorisation of the Ministry of Health $(\mathrm{MoH})$ in alignment with the National Treatment Guidelines and the National List for permitted medicines. While imported medicines have certificates of testing from manufacturers, few verifications and tests are performed on Bioko Island to determine if the imported medicines satisfy the $\mathrm{MoH}$ required compliance. As a systematic 


\section{Key questions}

\section{What is already known about this topic?}

- Early reports indicated that up to $35 \%$ of antimalarial medicines, purchased using non-representative sampling approaches) from 21 Sub-Saharan African countries had failed content analysis.

- In contrast, recent surveys to determine the quality of artemisinincontaining antimalarials (ACAs), the current first-line of treatment, purchased using systematic sampling approaches in five malariaendemic countries in Africa found less than $1 \%$ falsified ACAs (only in Nigeria), but that substandard ACAs were present in all countries.

\section{What are the new findings?}

- We used three different sampling approaches (convenience survey with mystery client, full island-wide survey with mystery client and randomised survey with overt sampling) to purchase 677 ACAs on Bioko Island, Equatorial Guinea.

- When analysed for content, we found $91.0 \%$ ACAs to be of acceptable quality (contained the stated active pharmaceutical ingredients in the correct concentration), $1.6 \%$ to be substandard and $7.4 \%$ falsified. No degraded medicines were detected.

- These findings substantiate previous reports of the presence of falsified Coartem in parts of West and Central Africa and provides additional evidence on the often overlooked problem of substandard medicines—-both of which render treatment ineffective.

\section{Recommendations for policy}

- These data underscore the vital need for national authorities to track the scale of ineffective medicines that jeopardise treatment of a life-threatening disease and the value of using a different sampling approach to understand the true prevalence of poor-quality medicines.

- The methods used on Bioko Island can be used by Ministries of Health to carry out regular surveillance to track the scale of substandard medicines in other countries. assessment of the quality of medicines had never been carried out in Equatorial Guinea, we conducted an island-wide survey of outlets to estimate the prevalence of poor-quality artemisinin-containing antimalarials (ACAs) on sale on Bioko Island, Equatorial Guinea.

\section{METHODS}

\section{The study area and sample collection}

Bioko Island, Equatorial Guinea, is one of the areas with the highest level of malaria transmission in the world. ${ }^{9}$ Bioko, which is the site of the country's capital Malabo, is the largest of the five islands of Equatorial Guinea with an area of $2017 \mathrm{~km}^{2}$ and population of about 250000 . Malaria transmission occurs year round, with an estimated entomological inoculation rate ranging from 163 to 840 in $2009,{ }^{10}$ which had decreased to 44 at the time of our study in 2013 according to the Bioko Island Malaria Control Project (BIMCP) reports. The fight against malaria in Equatorial Guinea is conducted via two initiatives supporting the National Malaria Control Programme (NMCP). In 2004, the BIMCP was launched in collaboration with the NMCP to combat the high malarial burden on the island. When the BIMCP began, island-wide prevalence of Plasmodium falciparum in children aged 2-14 years was $45 \%$. Through control measures, including the introduction of indoor residual spraying (IRS) and distribution of insecticide-treated nets (ITNs) to all households on Bioko Island, the prevalence has decreased to $27.8 \%$ in 2013 with a heterogeneous distribution, as seen in figure $1 \mathrm{~A}$ and $\mathrm{B}$.

\section{A}

Parasite Prevalence on Bioko Island in children 2-14 years 2013

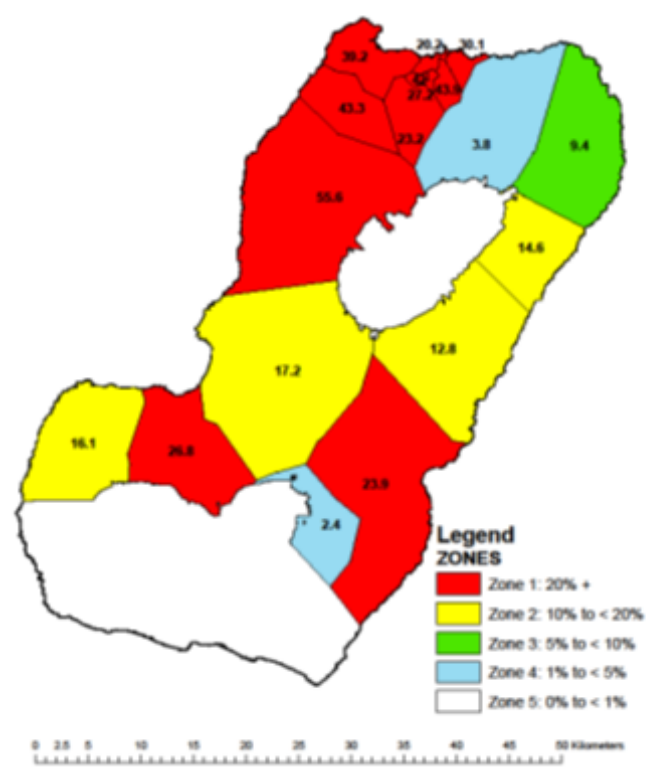
B

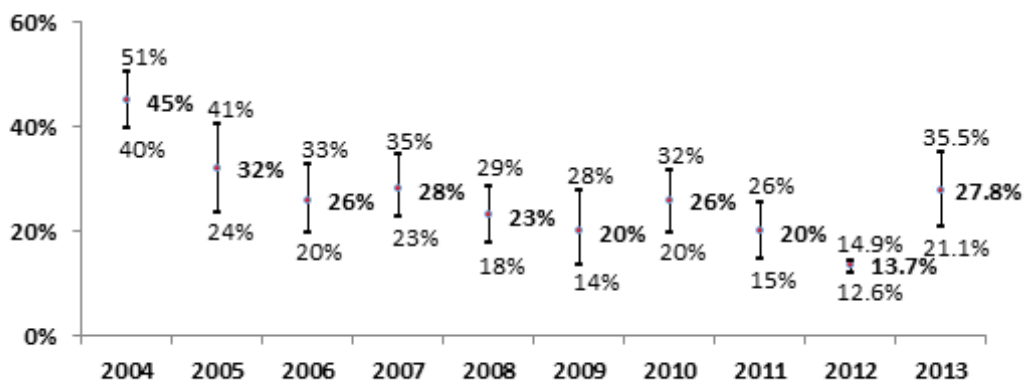

Figure 1 Map of Bioko Island stratified according to the parasite prevalence (A) and the prevalence of Plasmodium falciparum $(95 \% \mathrm{Cl})$ in 2-14 year olds on Bioko Island from 2004 to 2013 (B). 
In addition to IRS and ITNs, the BIMCP has supported the NMCP in providing the first-line ACAs free of charge since 2005. ACAs used in Equatorial Guinea have included artesunate plus sulfadoxine-pyrimethamine and artesunate plus amodiaquine. The latter was introduced in August 2008 as first-line treatment of uncomplicated malaria. However, while free of charge, ACTs are available on the island, many people do not choose to get medicines at public health centres and complain of side effects. ${ }^{11}$ The 2013 Malaria Indicator Survey revealed that only $38.6 \%$ of the interviewees who were sick in the past week and reported a positive malaria test, received treatment from the public health services, where medicines are free, while $55.7 \%$ sought their medicines at a private pharmacy.

A number of guidelines, including STROBE, ${ }^{12}$ MEDQUARG $^{13}$ and a recently published checklist of criteria for designing and reporting of medicine quality studies, ${ }^{14}$ informed the study design and analysis methods reported in this manuscript. In May 2013, the BIMCP conducted an island-wide census to identify both registered and unregistered drug providers, listing 278 outlets consisting of 180 pharmacies, 53 medicine vendors and 45 private clinics (see online supplementary table 1 ). Outlets were georeferenced, classified and included in the master database. Drug quality surveys were carried out at outlets listed in the census in three phases in June 2013. A team of 16 local surveyors worked in pairs to visit the outlets assigned to them by the supervisor, after receiving 2 days of training, including a 1-day pilot run to pretest the sample collection approaches.

Samples purchased included as many different brands and formulations as possible (i.e., blister packed tablets, granules, injections, capsules, syrups and suspensions, including age-specific dosage packages for the same brand). The minimum amount of each sample bought was defined as six individual units (i.e., six loose tablets, six ampoules for injection, six granule packs) or one bottle of the suspension. For blister packed tablets, 'one sample' was approximately one complete blister pack.

\section{Convenience survey using mystery client}

During the first phase (9 and 10 June 2013), outlets were purposely selected by the study team following the convenience survey with mystery client approach as recommended by United States Pharmacopeia, ${ }^{15}$ in an attempt to purchase as wide a variety of available medicines as possible. A total of 31 ACAs were purchased by 4 surveyors from 16 outlets which intentionally included providers that were suspected of selling outdated and unregistered products.

\section{Full island-wide survey using mystery client approach}

Bioko Island is small with relatively few licensed and unlicensed drug outlets; hence, it was feasible to visit them all. In the second phase (11-16 June 2013), all outlets open for business were visited by eight teams consisting of two surveyors per team, using a mystery client approach to purchase ACAs. The surveyors approached the provider using one of three predetermined scenarios where they feigned that they or a member of their family were sick and needed medications or that they needed to send antimalarials to relatives in the village. The surveyors acted as either a patient, a patient's friend or relative requesting to see all ACAs available before purchasing. A total of 174 outlets were visited and found to be open and selling ACAs.

\section{Randomised survey using overt sampling approach}

A simple randomised subset of 88 outlets (50.5\%) was drawn from a list of 174 eligible outlets (104 were excluded because they were either permanently closed or not selling antimalarials during the initial survey using the mystery clients). This third phase, undertaken by four teams of two surveyors each, was completed in 6 days (17-22 June 2013) and 278 ACAs were purchased. The provider was informed of the purpose of the drug purchase and their consent sought to participate in the study. A structured questionnaire was completed on the availability, supply and costs of the ACAs they sold. Providers were also questioned about their level of training, dispensing practices and storage conditions of medications.

\section{Sample processing}

At the end of each sample purchasing trip, the survey teams returned to the BIMCP offices in Malabo and completed an information form for every sample purchased recording the following information: the outlet type, date of purchase, the price paid, brand name, formulation, batch number, manufacture and expiry date. Each sample was placed in an individual zip-lock bag together with data recorded on their corresponding information forms and stored securely in an air-conditioned room $\left(\approx 20^{\circ} \mathrm{C}\right)$ pending dispatch to the London School of Hygiene and Tropical Medicine (LSHTM), London, UK via DHL Express, for further processing.

At LSHTM, digital photographs of packaging and contents were taken and sample information (brand name, SAPIs, dose form, outlet, district, date of purchase, name of stated manufacturer, country of manufacture, presence of the Affordable Medicines Facility-malaria, green leaf AMFm logo, batch number, date of manufacture, expiry date, number of tablets per blister packed and price paid) was checked with the information collected in the field and logged in a database using Epi Info V.3.5. ${ }^{16}$ Each tablet was weighed and its dimensions recorded prior to laboratory analysis.

\section{Laboratory SAPI content analysis}

For quantitative analysis by high-performance liquid chromatography with photodiode array (HPLC-PDA) detection, each pulverised tablet was combined with the appropriate solvent (see online supplementary table 2), sonicated and centrifuged. The supernatant, containing soluble active ingredients, was then injected into the 
HPLC column and the amount of SAPIs present in the tablet was determined. Other formulations (injections, granules, capsules, syrups and suspensions) were dissolved in methanol prior to HPLC analysis. HPLC analysis was conducted using a Dionex Ultimate 3000 system (Thermofisher, Hemel Hempstead, UK) and separation achieved using a GENESIS AQ $4 \mu \mathrm{m}$ column $(150 \times 4.6 \mathrm{~mm}$, Grace Materials Technologies, Cranforth, UK) or Acclaim 120, C18, 5 um Analytical $(4.6 \times 150 \mathrm{~mm})$ from Fisher Scientific, Leicestershire, UK. The mobile phase was a gradient of ammonium formate $(10 \mathrm{mM}, \mathrm{pH}$ 2.7 ), and acetonitrile (v/v; $15: 85$ to $85: 15$ over $7.0 \mathrm{~min}$ ) at the flow rate of $1.0 \mathrm{~mL} / \mathrm{min}$ was used to analyse all the formulations. Online supplementary table 2 outlines information of the solvents, columns, concentration injected and wavelengths set on the detector (DAD 3000) used to measure the SAPIs as well as the source of the reference standards for the HPLC analysis. A duplicate sample of the ones analysed at LSHTM from each packet were sent to the US Centres for Disease Control and Prevention (CDC) Laboratories in Atlanta, Georgia, USA for confirmatory HPLC analyses as well as to the Georgia Institute of Technology (GT), in Atlanta, Georgia, USA for ambient mass spectrometry (MS) analyses to verify the ingredients present and identify any unstated compounds. ${ }^{17} 18$ Each laboratory analysed the SAPIs in the combinations using different methodologies (HPLC columns and eluents). In general, there was very good agreement (mean bias was 1.16 with 95\% CI ranging from 15.4 to 17.8), between the results from the two laboratories where quantitation work was carried out (CDC and LSHTM) as shown by the Bland Altman plots (see online supplementary figure 1 ).

\section{Classification of samples}

The World Health Assembly recently agreed to adopt substandard, unregistered and falsified to define medicine quality. ${ }^{19}$ Where substandard refers to 'out of specification' medicines, these are authorised medical products that fail to meet either their quality standards or their specifications or both. 'Unregistered' defines any medical products that have not undergone evaluation and/or approval by the national or regional regulatory authority for the market in which they are marketed/distributed or used, subject to permitted conditions under national or regional regulation and legislation. 'Falsified' medical products deliberately/fraudulently misrepresent their identity, composition or source. Additional classifications are the degraded formulations that may result from exposure of good-quality medicines to light, heat and/or humidity. It is important to detect the products of degradation of SAPIs to distinguish degraded medicines from those that left the factory as substandard. ${ }^{20}$

Due to the lack of registration and verification of medicines entering Equatorial Guinea, it was not possible to determine if medicines in our study were unregistered or out of specification. Therefore, classification of medicines was solely based on their measured SAPI content and not on registration status. Laboratory results were expressed as a percentage of the SAPI on the packaging (\% SAPI). This was determined for both SAPIs including the artemisinin derivative and the associated partner drug, a non-artemisinin antimalarial. To accommodate the variation in HPLC laboratory testing of less than 10 samples of each ACA formulation, we adopted the tolerance range of $85 \%-115 \%$ for this study. ${ }^{21-23}$ If the \% SAPI of a drug was found to be outside of this tolerance band, then a second sample from the same packet was analysed and the average outcome reported (except suspensions as the entire bottle was used in the first analysis). The \% SAPI was used to classify each sample as one of the following: being of acceptable quality $(85 \%-115 \%$ for both SAPIs); substandard $(<85 \%$ or $>115 \%$ of either SAPI). Classification of analysed medicines as 'degraded' was achieved through comparison of HPLC retention times and MS patterns of good quality medicines with that obtained from artificially degraded samples. ${ }^{20}$ Degraded samples contained products of degradation of either and/ or both SAPIs. Artificial degradation was achieved by placing good quality artemether-lumefantrine (Coartem, Novartis, China) and artesunate-amodiaquine tablets (Winthrop, Maphar Laboratories, Morocco) in an oven at $60^{\circ} \mathrm{C}$ for 21 days. ${ }^{20}$ Samples were classified as falsified, when they were found to not contain the SAPIs, when they contained other compounds not listed on the packaging and when visible package differences were present after comparison with the packaging from acceptable quality medicines. Please note that packages from ACAs, where the SAPIs were found to comply with the pharmacopoeia tolerance limits (acceptable quality products), were used for comparison purposes, as authentic packaging could not be obtained from the manufacturers who hold the patents.

\section{Data analysis}

Laboratory data were combined with survey and sample data (S1 Data base) and analysed using STATA V.12 (Statacorp, College station, Texas, USA). Proportions were evaluated using $\chi^{2}$ tests, and crude associations between poor-quality ACAs and variables collected from the questionnaire were examined. A univariate logistic model assessed associations with poor-quality products and factors associated with a $\mathrm{p}<0.1$ on crude analysis were included in a multivariate model. The survey command (svy) of the software was used to compute clustering at the outlet level.

\section{Ethics approvals and findings reported to authorities}

The study was approved by the LSHTM Ethics Committee (Ref: 5804), and ethical approval from Equatorial Guinea's MoH was secured. This study did not involve patients, hence written consent was not required. Verbal consent was given by the person in charge of the outlet during the randomised-overt survey sampling approach which was recorded on the database. Findings were reported to the $\mathrm{MoH}$ on Bioko Island; manufacturers of medicines found 
to be falsified; Global Fund drug quality section and the WHO (rapidalert@who.int).

\section{RESULTS}

Samples of ACAs ( $\mathrm{n}=677)$ consisting of artemisinin monotherapies (AMTs) and ACTs were purchased from 278 outlets selling antimalarial medicines, located mainly in the capital, Malabo $(64.7 \%$ pharmacies, $19.1 \%$ medicine vendors and $16.2 \%$ private clinics; see online supplementary table 1). SAPI content analyses were carried out in three independent laboratories to provide robust estimates of the prevalence of acceptable quality, substandard, falsified and degraded AMTs and ACTs. Overall, the majority $(64.7 \%)$ of pharmacies and private clinics (without inpatient capacity) had at least one ACA available at the time of the randomised-overt survey, while only about a quarter of the medicine vendors had an ACA available (see online supplementary table 1 ). The medicine vendors included a diverse group of outlets: some of them sell various household items, including medicines, while others sell only medicines either in a small shop or just inside their house, in a clandestine way. Medicine vendors and private clinics were more likely than pharmacies to have staff with a health-related qualification and/ or recent malaria training (past 12 months); however, pharmacies on average stocked almost four times as many ACA samples of varying brands than the medicine vendors.

Table 1 shows the quality of ACAs purchased using the various sampling strategies. The convenience survey using mystery client yielded 17 ACTs and 14 AMTs, representing 12 different brands, purchased from 16 outlets. Of these, $80.7 \%$ were of acceptable quality (containing 85-115 for both SAPIs), 3.2\% substandard and $16.1 \%$ were falsified that did not contain the SAPIs but contained unstated compounds that were not listed on the packets. The full island-wide survey using mystery client yielded 60 brands purchased from 174 outlets (368 samples; 196 ACTs and 172 AMTs). Among these, 91.9\% were acceptable quality medicines, $5 \%$ substandard and $7.6 \%$ were falsified. The randomised survey using overt sampling approach found 132 ACTs and 146 AMTs, 64 brands purchased from 88 outlets. From these samples, $91.0 \%$ were of acceptable quality, $2.9 \%$ substandard and $6.1 \%$ were falsified. No degraded samples were detected among any of the medicines purchased on Bioko Island, suggesting that the duration and conditions of storage of the medicines were adequate.

Table 1 Chemical analyses of antimalarial medicines purchased using three approaches (convenience-mystery clients, full island-wide survey-mystery clients and randomised-overt sampling) on Bioko Island, Equatorial Guinea; $\mathrm{n=677}$

\begin{tabular}{|c|c|c|c|c|c|}
\hline Outlets (n) & Acceptable Quality AD and PD & Substandard & Degraded & Falsified & Tota \\
\hline \multicolumn{6}{|c|}{ Convenience survey using mystery client (samples=31; total brands=12; brands per outlet=0.8) } \\
\hline Pharmacies (10) & $16(76.2 \%)$ & $1(4.8 \%)$ & 0 & $4(19.0 \%)$ & 21 \\
\hline Medicine vendors (6) & $9(90.0 \%)$ & 0 & 0 & $1(10.0 \%)$ & 10 \\
\hline Private clinics $(0)$ & 0 & 0 & 0 & 0 & 0 \\
\hline All outlets (16) & $25(80.7 \%)^{*}$ & $1(3.2 \%)$ & 0 & $5(16.1 \%)^{\star \star \star}$ & 31 \\
\hline \multicolumn{6}{|c|}{ Full island-wide survey using mystery client (samples=368; total brands=60; brands per outlet=0.3) } \\
\hline Pharmacies (108) & $236(91.1 \%)$ & $2(0.8 \%)$ & 0 & $21(8.1 \%)$ & 259 \\
\hline Medicine vendors (33) & $50(92.6 \%)$ & 0 & 0 & $4(7.4 \%)$ & 54 \\
\hline Private clinics (33) & $52(94.5 \%)$ & 0 & 0 & $3(5.5 \%)$ & 55 \\
\hline All outlets (174) & $338(91.9 \%)^{*}$ & $2(0.5 \%)^{\star \star}$ & 0 & $28(7.6 \%)$ & 368 \\
\hline \multicolumn{6}{|c|}{ Randomised-overt survey(samples=278; total brands=64; brands per outlet=0.7) } \\
\hline Pharmacies (62) & $213(90.2 \%)$ & $7(3.0 \%)$ & 0 & $16(6.8 \%)$ & 236 \\
\hline Medicine vendors (14) & $8(100.0 \%)$ & 0 & 0 & 0 & 8 \\
\hline Private clinics (12) & $32(94.2 \%)$ & $1(2.9 \%)$ & 0 & $1(2.9 \%)$ & 34 \\
\hline All outlets (88) & $253(91.0 \%)$ & $8(2.9 \%)^{* *}$ & 0 & $17(6.1 \%)^{\star \star \star}$ & 278 \\
\hline
\end{tabular}

Significant difference between sampling approaches for each quality category was as follows:

${ }^{*} \mathrm{p}=0.037$ : between convenience survey using mystery clients and full island-wide survey using mystery clients for acceptable quality (AD and $\mathrm{PD})$ samples.

${ }^{* *} \mathrm{p}=0.017$ : between full island-wide survey using mystery clients and randomised-overt survey for substandard samples.

${ }^{* * *} \mathrm{p}=0.040$ : between convenience survey using mystery clients and randomised-overt survey for falsified samples.

Other comparisons were not significantly different $(p>0.050)$

Acceptable quality: $85 \%-115 \%$ SAPIs for both AD and PD.

Substandard: $<85 \%$ or $>115 \%$ SAPI of either AD or PD or both.

Degraded: $<85 \%$ SAPI plus products of degradation of either/both AD or PD or both.

Falsified: zero SAPI for both AD and PD and visible differences (mistakes) on the packaging.

$A C A$, artemisinin-containing antimalarial; $A D$, artemisinin derivative (always the ACA); PD, partner drug (always the non-ACA); SAPI, stated active pharmaceutical ingredient. 
Table 2 Quality of artemisinin-containing antimalarials at 76 outlets visited during both the island-wide survey using mystery client and the randomised-overt survey on Bioko Island, Equatorial Guinea

\begin{tabular}{llll}
\hline & \multicolumn{2}{c}{ Sampling method } & \\
\cline { 2 - 3 } Variable & Island-wide survey-mystery client & Randomised-overt survey & p Value \\
\hline Outlets & 76 of $174(43.7 \%)$ & 76 of $88(86.4 \%)$ & \\
Samples & 170 & 245 & $0.0057^{*}$ \\
Brands & $44(64.7 \%)$ & $58(85.3 \%)$ & 0.45 \\
Authentic & $154(90.6 \%)$ & $227(92.7 \%)$ & 0.35 \\
Substandard & $2(1.2 \%)$ & $6(2.4 \%)$ & \\
Degraded & 0 & 0 & 0.17 \\
\hline Falsified & $14(8.2 \%)$ & $12(4.9 \%)$ & \\
\hline
\end{tabular}

Total number of brands purchased $=68$.

*Significant difference.

All samples were analysed using MS to confirm the presence or absence of the SAPI and confirmed the HPLC results. Compounds present in falsified formulations identified by MS included bis(2-ethylhexyl) adipate, dioctyl adipate, chlorzoxazone, ciprofloxacin, 2-mercaptobenzothiazole, sildenafil, stearic acid, a disaccharide and a polymer of sugar alcohol (see online supplementary table 3). All but four of these unstated compounds were detected among samples collected by at least two of the different sampling approaches.

A significantly higher proportion of falsified medicines were found in samples purchased using the convenience survey compared with the randomised-overt sampling (table $1,16.1 \%$ vs $6.1 \%, \mathrm{p}=0.040$ ); Convenience mystery client approach also yielded a lower proportion of ACAs of acceptable quality than the island-wide survey-mystery client approach $(80.7 \%$ vs $91.9 \%$ respectively, $\mathrm{p}=0.037)$.

\section{Data on medicine quality}

Direct comparison of the full island-wide survey using mystery client and randomised survey using overt sampling approaches showed that, for a subset of 76 outlets that were visited during both sampling approaches, a greater number of samples and significantly more brands were collected using the randomised-overt approach (samples: 245 and 170: 58 and 44, respectively; table 2). There were no significant differences in the proportion of poorquality medicines purchased using these two sampling methods ( $p>0.05$ in all cases).

In all three sampling approaches, approximately half of the medicines purchased were ACTs (345) and the other half were AMTs (332), which included monotherapy suspensions (7), injections (129), capsule (1) and blister packed tablets (195). The availability of artesunate monotherapy tablets differed significantly according to sampling approach (convenience-mystery client $=45.2 \%$; full island-wide survey-mystery client $=32.6 \%$; randomisedovert sampling approach $=21.9 \%$; table 3 ) and 23 out of 62 blister packed tablets $(37.1 \%)$ of artesunate were found not to contain the SAPI. No AMTs of dihydroartemisinin were found among the purchased samples. Half of the samples that were found not to contain the SAPIs $(\mathrm{n}=50)$, purchased during all three sampling approaches were stated to be manufactured by WHO prequalified companies (table 4).

\section{Packaging examination}

We did not succeed in obtaining authentic samples of medicines and packaging from manufacturers who hold the patents. Hence, detailed investigations of the packaging were conducted between acceptable quality $(85 \%-$ $115 \%$ for both SAPIs) and substandard medicines, $(<85 \%$ of SAPIs) to check for identifiable visible differences, including signs of tampering, that is, deliberate change of batch number, manufacturing or expiry dates. There were no visible differences in the packaging between the acceptable quality and substandard medicines as well as no obvious indications that they had been manufactured by anyone other than the stated manufacturer.

In contrast, packaging associated with samples that were found not to contain the SAPIs but contained unstated compounds, and therefore classified as falsified, showed noticeable differences to packaging of acceptable quality medicines. For example, the falsified package for Artesunat (see online supplementary scan 1) had misspellings, a different mosquito, a different hologram and a different registration number as reported previously. ${ }^{8}$ All of the writing on the acceptable quality packets of Vatunate artesunate is in green ink whereas the falsified packets are in black ink, the front of the packet is halfgreen and half white (see online supplementary scan 2). Comparison of packets of Coartem showed that the falsified product had a 24-month time period between the manufacturing and expiry dates whereas the manufacturer, Novartis, confirmed that it should be 23 months. Additionally, the backs of the blister packed fully covered with cardboard circles whereas the acceptable quality product has half circles of cardboard (see online supplementary scan 3). Package containing falsified tablets of dihydroartemisinin-piperaquine packaged as Duo-cotecxin had a stamp stating, 'donated by P R China', and the colours on the packets are faded while the lot 


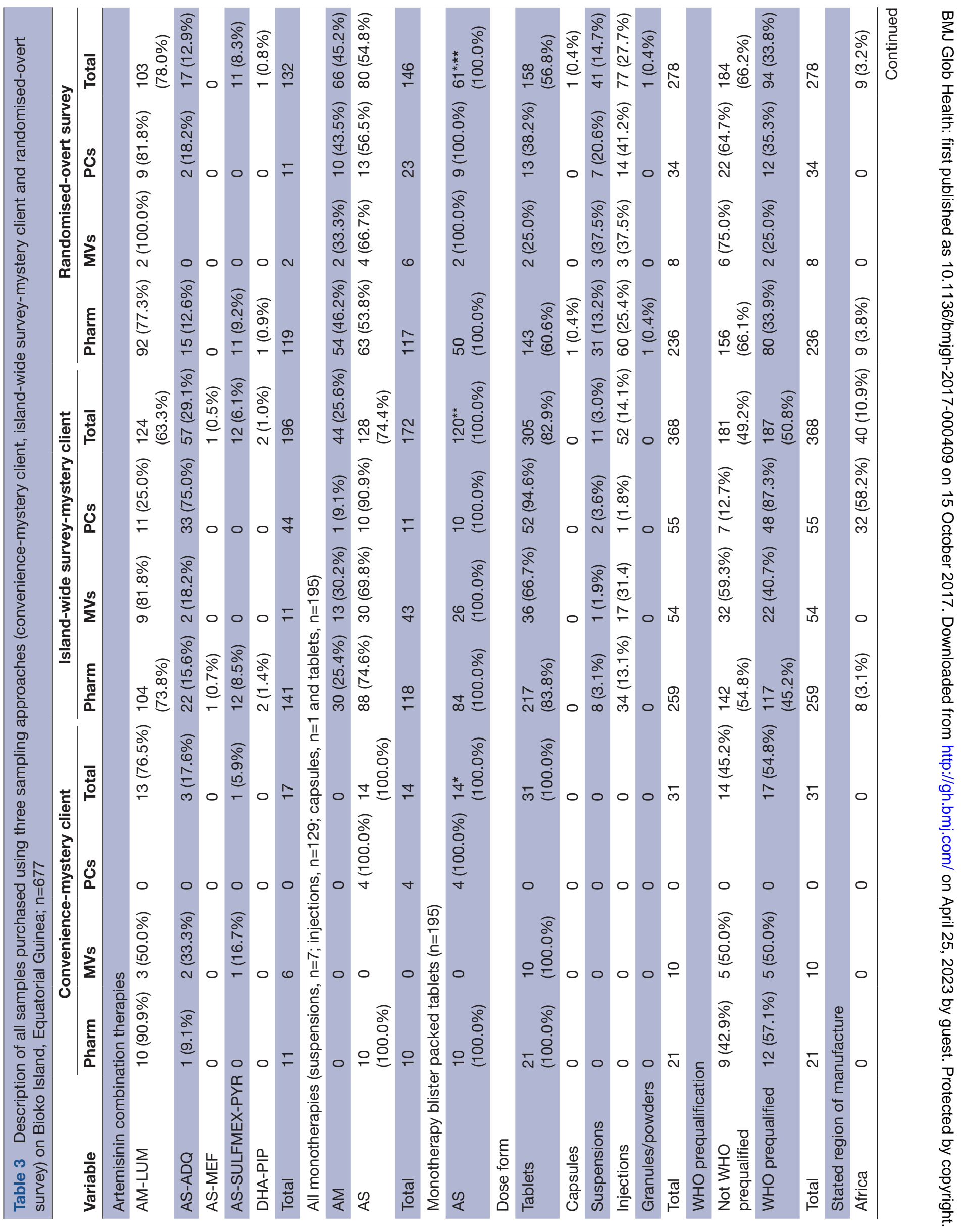




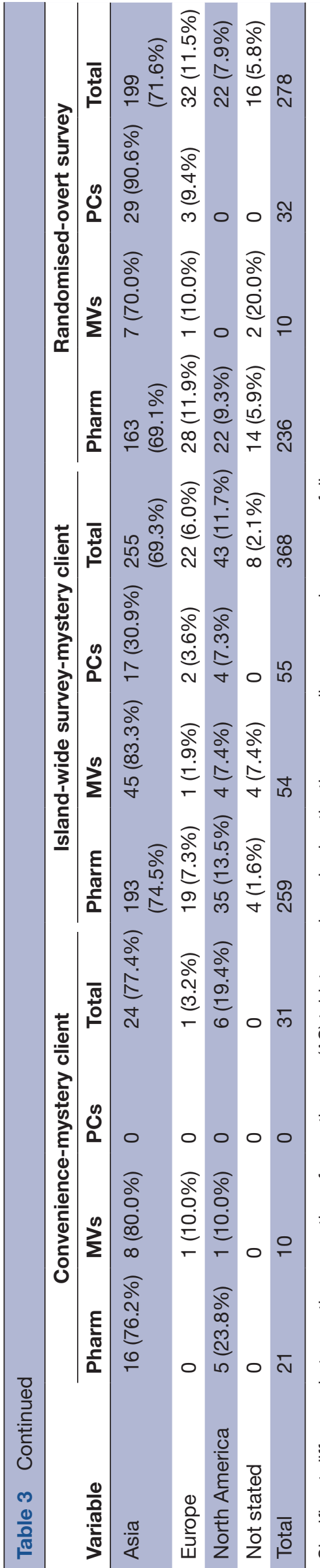

number, manufacturing and expiry date are missing (see online supplementary scan 4).

\section{Risk factors for substandard, degraded and falsified medicines}

There was no significant difference in the drug quality among the artemether, artesunate and dihydroartemisinin formulations nor between samples of medicines produced by WHO prequalified manufacturers compared with those from manufacturers that are not prequalified (table 4). At the time of the laboratory analysis, all but five samples were within their expiry dates of which one packet of tablets was falsified and the four suspensions were substandard. Among pharmacies, a slightly higher proportion of the ACAs sampled were of poor quality, compared with ACAs purchased from medicine vendors or private clinics, though the risk was not significantly increased ( $p=0.14$, table 4$)$. In contrast, samples labelled with the AMFm logo were more likely to be of poor quality than those that were not $(\mathrm{p}<0.001)$. In addition, poor-quality medicines were associated with stated manufacture in North America $(p<0.001)$. These findings are likely to be influenced by the large number of falsified Coartem samples that were in circulation. Approximately $50 \%$ (26 out of 54) of the Coartem purchased were found to be falsified (see online supplementary table 4). Five variants of falsified Coartem were identified (batch numbers F1901, F2261, F2596 and NOF2153), all allegedly manufactured by Novartis and with the AMFm logo, with one exception (F1901), yet containing a range of various compounds instead of the SAPIs. In addition to the falsified Coartem, $86 \%$ (19 of the 22) of the Artesunat monotherapy samples purchased were found to be falsified.

\section{DISCUSSION}

Poor-quality ACAs are postulated to cause a serious direct health risk to consumers and several studies have focused on the impact of falsified medicines on public health. ${ }^{1} 78$ 21-23 Recently, additional concern has been raised over the possibility that substandard antimalarials could lead to drug resistance as a result of underdosing, though the association has yet to be proven. ${ }^{24}$ Identifying the scale of substandard and falsified ACAs in the market place in malaria-endemic countries is an essential first step to address this impediment in the fight against malaria.

Over $90 \%$ of samples of ACTs and AMTs (n=677, 142 brands) purchased from pharmacies, medicine vendors and private clinics on Bioko Island were found to be of acceptable quality (contained the specified SAPIs). The poor-quality medicines consisted of substandard (1.6\%) and falsified ACAs (7.4\%), where the latter contained compounds other than the SAPIs. No degraded medicines were detected indicating either storage in the absence of high heat and humidity and/or a fast turnover of medicines. The reliability of these findings is supported by the 


\begin{tabular}{|c|c|c|c|c|c|}
\hline \multicolumn{2}{|c|}{$\begin{array}{c}\text { Sample characteristics } \\
\text { of ACAs }\end{array}$} & \multirow{2}{*}{$\begin{array}{l}\text { Total number } \\
\text { of samples } \\
350\end{array}$} & \multirow{2}{*}{$\begin{array}{l}\text { Poor-quality } \\
\text { samples }\end{array}$} & \multirow{2}{*}{$\begin{array}{l}\text { Crude ORs } \\
(95 \% \mathrm{Cl})\end{array}$} & \multirow{2}{*}{$\begin{array}{l}\text { Likelihood- } \\
\text { ratio p value } \\
0.36\end{array}$} \\
\hline Generic type & AM & & & & \\
\hline & DHA & 3 & 1 (33.3\%) & 4.6 (0.4 to 52.6$)$ & \\
\hline & AS & 324 & $26(8.0 \%)$ & 0.8 (0.5 to 1.4$)$ & \\
\hline \multirow{2}{*}{$\begin{array}{l}\text { WHO prequalified/ } \\
\text { AQACT }\end{array}$} & Not prequalified & 379 & $35(9.2 \%)$ & 1 & 0.82 \\
\hline & Prequalified & 298 & $26(8.7 \%)$ & $0.9(0.6$ to 1.6$)$ & \\
\hline \multirow[t]{2}{*}{ AMFm } & Non-AMFm medicines & 579 & $36(6.2 \%)$ & 1 & $<0.001$ \\
\hline & AMFm medicines & 98 & $25(25.5 \%)$ & 5.2 (2.9 to 9.1$)$ & \\
\hline \multirow{3}{*}{$\begin{array}{l}\text { Region of stated country } \\
\text { of manufacture }\end{array}$} & Asia & 471 & $33(7.0 \%)$ & 1 & $<0.001$ \\
\hline & North America & 71 & 26 (36.6\%) & 7.7 (4.2 to 14.0$)$ & \\
\hline & Not stated & 73 & $2(2.7 \%)$ & $0.4(0.1$ to 1.6$)$ & \\
\hline \multirow{2}{*}{$\begin{array}{l}\text { Expired at time of } \\
\text { analysis }\end{array}$} & Not expired & 663 & $56(8.4 \%)$ & 1 & $<0.001$ \\
\hline & Expired $^{*}$ & 14 & $5(35.7 \%)$ & 6.0 (2.1 to 17.0$)$ & \\
\hline \multirow[t]{3}{*}{ Outlet type } & Pharmacies & 519 & $51(9.8 \%)$ & 1 & 0.14 \\
\hline & Medicine vendors & 75 & $5(6.7 \%)$ & 0.7 (0.3 to 1.7$)$ & \\
\hline & Private clinics & 89 & $5(5.6 \%)$ & 0.5 (0.2 to 1.4$)$ & \\
\hline
\end{tabular}

${ }^{*}$ Of the five expired samples, one was a falsified tablet and four were substandard suspensions.

ACAs, artemisinin-containing antimalarials; AM, artemether; AMFm, Affordable Medicines Facility-malaria; AQACT, acceptable qualityartemisinin combination therapy; AS, artesunate; DHA, dihydroartemisinin; ORs, odds ratio.

use of standardised sampling procedures to purchase the samples followed with analysis at three independent laboratories. Laboratory analysis of samples using both HPLC-PDA and MS techniques confirmed the absence of the SAPIs and the presence of alternative compounds, none of which have antimalarial properties.

Packets of artesunate monotherapies were available on Bioko, despite these products not being recommended by WHO for the treatment of uncomplicated falciparum malaria nor included in the NMCP treatment guidelines for Equatorial Guinea, as monotherapies are considered to be a major contributing factor to the development of artemisinin resistance. ${ }^{25}$ Of additional concern is that $37.1 \%$ of artesunate monotherapy samples analysed were falsified, that is, did not contain the SAPI and had noticeable differences in the packaging from that of acceptable quality samples. This is of particular relevance given the first reported case of falsified artesunate monotherapy, which almost resulted in a fatality, was purchased on the Equatorial Guinea mainland. ${ }^{8}$ Both the absence of one or both of the SAPI in an ACT or AMT lead to absence of drug efficacy, which can cause dangerous effects/ events. Furthermore, the availability of monotherapies can increase the risk of drug resistance. ${ }^{26}$

Our results are reassuring in that samples labelled with the AMFm logo or from WHO prequalified manufacturers were less likely to have concerns related to medicine quality than those without these accreditations. However, there is still a need for vigilance with these products, as highlighted by samples of falsified Coartem 20/120 (batch F2261) bearing the AMFm logo originally and allegedly manufactured by Novartis, that were originally sold in Angola 2728 and subsequently purchased in Enugu metropolis, Nigeria ${ }^{21}$ and Bioko Island (current study). These particular batches of Coartem were also reported by Novartis to be found in Cameroon. ${ }^{29}{ }^{30}$ Relying solely on packaging appearance to detect falsified medicines may not be sufficient, and results in this study suggest that using devices that have the potential to detect the chemical quality of drugs at the point of purchase should be used. A low-cost, hand-held near-infrared spectrometer device marketed at currently USD 250 has recently been demonstrated to be effective in identifying the falsified samples of Coartem across multiple batches and manufacturing locations. ${ }^{31}$ Such low-cost screening devices could greatly facilitate the ability of regulatory authorities to screen the quality of medicines as well as identify suspect products in need of confirmatory pharmacopoeia methods such as HPLC, regarded as the gold standard.

When comparing the estimates obtained using different sampling approaches, the prevalence of falsified medicines was higher for ACAs purchased using the convenience survey-mystery client approach compared with the island-wide mystery client and random survey-overt sampling approaches. These findings are not surprising as the outlets chosen in the convenience mystery client approach were selected based on local knowledge to include outlets known to sell expired medicines, medicines banned in other countries and/or medicines without proper labelling, thus increasing the probability that they also had falsified antimalarials. A previous study in Enugu, Nigeria $(n=3024)$ similarly noted a higher proportion of falsified samples were purchased when 
using a convenience mystery client approach $(3.0 \%)$ versus a full survey using mystery client or random-overt sampling approach $\left(1.2 \%\right.$ and $0.6 \%$, respectively) ${ }^{21}$ In contrast, in both studies, there was no significant difference in the quality of the medicines purchased using the mystery shopper or overt sampling approach. Together, these findings indicate that either sampling strategy could be used effectively to determine drug quality in a geographical region. However, as each approach has its own advantages and disadvantages, the survey objectives need to be considered to ensure that the most appropriate sampling strategy is chosen..$^{21}$ For example, overt sampling has the advantage of being able to collect additional information using outlet questionnaires, enabling detailed data collection on sources as well as risk factors for poor-quality medicines, at minimal added cost. Our findings also indicate that it may be easier to collect a greater variety of medicines at each outlet using an overt sampling approach rather than mystery shopper. However, as outlet staff are aware that a survey is taking place, there is a risk that they may withhold known poorquality medicines from the survey team. While this study did not indicate that this sampling bias was present based on the percentage of falsified medicines, fewer monotherapy treatments, which are not in the country's National Treatment guidelines, were collected during the overt sampling. The mystery client approach has the advantage of a lower inherent risk of sampling bias as outlets are unaware of the survey. However, as shown in our study, it is difficult to obtain as many samples from each outlet using this approach. In this study, both the island-wide survey using mystery client and the randomised-overt sampling approaches yielded similar results for each classification of drug quality (acceptable, substandard and falsified while no degraded samples were found) indicating that the selection bias often assumed to be the limitation of overt surveys was not a major concern in this setting. This would further suggest that in contrast to monotherapies which are readily identifiable, vendors are not necessarily aware of which medicines may be of poor quality. While the packaging of falsified medicines in this study had noticeable differences (spelling, logo, differences in colour and so on) to those from acceptable quality medicines, these differences may not be obvious to the untrained eye. Laboratory analysis of samples using both HPLC-PDA and MS techniques confirmed the absence of the SAPIs and the presence of alternative compounds, none of which has antimalarial properties.

Our results from Bioko Island show that Coartem and artesunate monotherapy tablets are most likely to be falsified in this setting. The risk of purchasing a falsified drug was greater from a pharmacy than other sources. Yet only one-third of the pharmacies in the census were officially registered with the $\mathrm{MoH}$, highlighting the challenge to be faced in obtaining reliable estimates of drug quality in settings where regulation is weak and surveys hampered by factors such as lack of sampling frame, rapid turnover of outlets, providers fearing regulation and/or reprisal, staff safety and anonymity. Nevertheless, in many countries, a major share of antimalarial treatments are obtained from unregulated providers, and drug quality in these outlets needs to be a priority for research. Our study shows that carrying out a census of pharmacies in the area before conducting a survey, and not just relying on a list of registered pharmacies from the $\mathrm{MoH}$, can help identify unregistered outlets to be included in the sampling frame.

\section{CONCLUSION}

Our data show how a convenience survey using mystery client can be useful to identify 'hot spots' of poor-quality medicines, but that a representative survey based on a defined sampling frame, and using either mystery client or an overt approach to purchase drugs, is needed to accurately quantify and track the scale of ineffective medicines. Our investigation did not find the prevalence of poor-quality medicines on Bioko Island to be as alarming as reported elsewhere in Sub-Saharan Africa, ${ }^{32-34}$ but we did find ACA medicines that did not contain the SAPIs and were in falsified packaging. The findings from Bioko Island reinforce those from our previous study in Enugu, Nigeria and highlight the need for routine monitoring of medicine quality, following a representative sampling approach, to ensure that the available treatments for life-threatening diseases are not jeopardised.

\section{Author affiliations}

${ }^{1}$ Department of Clinical Research, London School of Hygiene and Tropical Medicine, London, UK

${ }^{2}$ Division of Parasitic Diseases and Malaria, US Centers for Disease Control and Prevention, Atlanta, Georgia, USA

${ }^{3}$ Division of laboratory Sciences, Organic Analytical Toxicology Branch, Centers for Disease Control and Prevention, Atlanta, Georgia, USA

${ }^{4}$ Facultad de Bioquímica y Ciencias Biológicas, Universidad Nacional del Litoral, and Consejo Nacional de Investigaciones Científicas y Técnicas (CONICET), Santa Fe, Argentina

${ }^{5}$ School of Chemistry and Biochemistry, Georgia Institute of Technology, Atlanta, Georgia, USA

${ }^{6}$ Bioko Island Malaria Control Project, Medical Care Development International, Malabo, Equatorial Guinea

Acknowledgements Special thanks are accorded to the following: Surveyors for their discretion and participation in purchasing the samples in this study; Jamie Long for his assistance with the laboratory analyses of the samples; Julian Robinson for independently examining the matched substandard and acceptable quality sample packaging. Siân Clarke for her comments on the manuscript.

Contributors HK and FM conceived the study. ZH developed methodology to analyse for the degradation products. GG, DH and IM carried out fieldwork and data collections. HK, ELA, ZH, MDG, IS, FMF, PD and MJC performed HPLC-PDA and MS analysis. IM and ELA carried out the statistical analyses of the data. HK wrote the first draft of this manuscript and FM, GG and DH provided all local information. All authors participated in the revision until production of the final draft.

Funding The authors are grateful to the Bill and Melinda Gates Foundation for funding the staff salary and HPLC analysis costs as part of an award to the ACT consortium at the London school of hygiene \& tropical medicine. Ministry of Health and Social Welfare, Malabo, Equatorial Guinea (through Medical Care Development International Bioko Island Malaria Control Project) are thanked for funding the sample purchase on Bioko Islands, Equatorial Guinea. The funders had no role in study design, data collection and analysis, decision to publish or preparation of the manuscript.

Competing interests None declared. 
Provenance and peer review Not commissioned; externally peer reviewed.

Data sharing statement No additional data are available.

Open Access This is an Open Access article distributed in accordance with the Creative Commons Attribution Non Commercial (CC BY-NC 4.0) license, which permits others to distribute, remix, adapt, build upon this work non-commercially, and license their derivative works on different terms, provided the original work is properly cited and the use is non-commercial. See: http://creativecommons.org/ licenses/by-nc/4.0/

(C) Article author(s) (or their employer(s) unless otherwise stated in the text of the article) 2017. All rights reserved. No commercial use is permitted unless otherwise expressly granted.

\section{REFERENCES}

1. Nayyar GM, Breman JG, Herrington JE. The global pandemic of falsified medicines: laboratory and field innovations and policy perspectives. Am J Trop Med Hyg 2015;92(6 Suppl):2-7.

2. Chaccour C, Kaur H, Del Pozo JL. Falsified antimalarials: a minireview. Expert Rev Anti Infect Ther 2015;13:1-5.

3. Who health organization http://www.who.int/features/factfiles/ malaria/en (accessed 15 May 2017).

4. Who health organization http://www.who.int/malaria/areas/ treatment/overview/en/- (accessed 12 May 2017).

5. White NJ. Can new treatment developments combat resistance in malaria? Expert Opin Pharmacother 2016;17:1303-7.

6. Who health organization. Antimalarial drug efficacy and drug resistance. http://www.who.int/malaria/areas/treatment/drug_ efficacy/en (accessed 17 Jul 2017).

7. Newton PN, Green MD, Mildenhall DC, et al. Poor quality vital antimalarials in Africa - an urgent neglected public health priority. Malar J 2011;10:352.

8. Chaccour CJ, Kaur H, Mabey D, et al. Travel and fake artesunate: a risky business. Lancet 2012;380:1120.

9. Noor AM, Kinyoki DK, Mundia CW, et al. The changing risk of Plasmodium falciparum malaria infection in Africa: 2000-10: a spatial and temporal analysis of transmission intensity. Lancet 2014;383:1739-47.

10. Overgaard HJ, Reddy VP, Abaga S, et al. Malaria transmission after five years of vector control on Bioko Island, Equatorial Guinea. Parasit Vectors 2012;5:253.

11. Patient information leaflet https://extranet.who.int/prequal/sites/ default/files/documents/MA057Part3v2.pdf(accessed 11 Jul 2017).

12. von Elm E, Altman DG, Egger M, et al. Strengthening the Reporting of Observational Studies in Epidemiology (STROBE) statement: guidelines for reporting observational studies. BMJ 2007;335:806-8

13. Newton PN, Lee SJ, Goodman C, et al. Guidelines for field surveys of the quality of medicines: a proposal. PLoS Med 2009;6:e1000052.

14. Lalani M, Kitutu FE, Clarke SE, et al. Anti-malarial medicine quality field studies and surveys: a systematic review of screening technologies used and reporting of findings. Malar J 2017;16:197.

15. United States Pharmacopeia-Guidelines for sampling of antimalarial drug samples in the USP DQ1. Antimalarial drug quality monitoring project in mekong sub-region countries. $2001 \mathrm{http}: / /$ pdf. usaid.gov/pdf_docs/PNADH150.pdf- (accessed 9 Feb 2012).
16. http://epi-info.software.informer.com/- (accessed 18 Jul 2016).

17. Monge ME, Harris GA, Dwivedi P, et al. Mass spectrometry: recent advances in direct open air surface sampling/ionization. Chem Rev 2013;113:2269-308.

18. Culzoni MJ, Dwivedi P, Green MD, et al. Ambient mass spectrometry technologies for the detection of falsified drugs. Med. Chem. Commun. 2014;5:9-19.

19. Who health Organization http://www.who.int/medicines/regulation/ ssffc/definitions/en/-(accessed 10 Jul 2017).

20. Hall Z, Allan E, van Schalkwyk D, et al. Degradation of artemisininbased combination therapies under tropical conditions. Am J Trop Med Hyg 2016;95-.

21. Kaur H, Allan EL, Mamadu I, et al. Quality of artemisinin-based combination formulations for malaria treatment: prevalence and risk factors for poor quality medicines in public facilities and private sector drug outlets in Enugu, Nigeria. PLoS One 2015;10:e0125577.

22. ACT Consortium Drug Quality Project Team and the IMPACT2 Study Team. Quality of Artemisinin-Containing Antimalarials in Tanzania's Private Sector--Results from a Nationally Representative Outlet Survey. Am J Trop Med Hyg 2015;92:75-86.

23. Yeung S, Lawford HL, Tabernero P, et al. Quality of antimalarials at the epicenter of antimalarial drug resistance: results from an overt and mystery client survey in Cambodia. Am J Trop Med Hyg 2015;92(6 Suppl):39-50.

24. Newton PN, Caillet C, Guerin PJ. A link between poor quality antimalarials and malaria drug resistance? Expert Rev Anti Infect Ther 2016;14:531-3.

25. Who health organization http://www.who.int/malaria/areas/ treatment/withdrawal_of_oral_artemisinin_based_monotherapies/ en/-(accessed 12 Jul 2017)

26. Ashley EA, Dhorda M, Fairhurst RM, et al. Spread of artemisinin resistance in Plasmodium falciparum malaria. $N$ Engl $\mathrm{J}$ Med 2014;371:411-23.

27. Faucon B, Murphy C, Whalen J. Africa's Malaria Battle: Fake Drug Pipeline Undercuts Progress. Wall Street Journal 2013 http://onli ne.wsj.com/article/SB1000142412788732447400457844494284 1728204.html (accessed 15 May 2017).

28. Newton PN, Tabernero P, Dwivedi $P$, et al. Falsified medicines in Africa: all talk, no action. Lancet Glob Health 2014;2:e509-e510.

29. Novartis: coartem malaria drugs. https://thecounterfeitreport.com/ product/395/Novartis-Coartem-Malaria-Drugs.html- (accessed 19 May 2016).

30. Falsified batches of Coartem recently circulating in Cameroon. http://www.who.int/medicines/publications/drugalerts/Alert_130 Information_Coartem_VF.pdf?ua=1 (accessed 10 Jul 2017).

31. Wilson BK, Kaur H, Allan EL, et al. Demonstration of a new handheld device for the detection of falsified medicines - detection of falsified artemisinin-based combination therapies. Am J Trop Med Hyg. May 2017;96:1117-23.

32. Tabernero P, Fernández FM, Green M, et al. Mind the gaps-the epidemiology of poor-quality anti-malarials in the malarious world--analysis of the WorldWide Antimalarial Resistance Network database. Malar J 2014;13:139.

33. Nayyar GM, Breman JG, Newton PN, et al. Poor-quality antimalarial drugs in southeast Asia and sub-Saharan Africa. Lancet Infect Dis 2012;12:488-96.

34. Roberts M. Third of malaria drugs 'are fake'. 2012 www.bbc.co.uk/ news/health-18147085 (accessed 12 May 2017). 